FIG. 2 Three successive rounds of DNA shuffling were done and the cells were grown on increasing cefotaxime levels. The MIC of cefotaxime (Sigma) for E. coli XL1-blue (Stratagene, San Diego) carrying wild-type p182Sfi is $0.02 \mu \mathrm{g} \mathrm{ml}^{-1}$. A mutant with a 16,000 -fold increased resistance to cefotaxime was obtained $(\mathrm{MIC}=$ $320 \mu \mathrm{g} \mathrm{ml}^{-1}$ ). This mutant was backcrossed twice, by shuffling with a 40 -fold excess of wildtype DNA. The backcrossed mutant was $32,000-$ fold more resistant than the wild type $\left(\mathrm{MIC}=640 \mu \mathrm{g} \mathrm{ml}^{-1}\right)$. After selection, the plasmid of selected clones was transferred back into wild-type $\mathrm{XL}-1$ blue cells to ensure that none of the measured drug resistance
Shuffling

Backcrossing

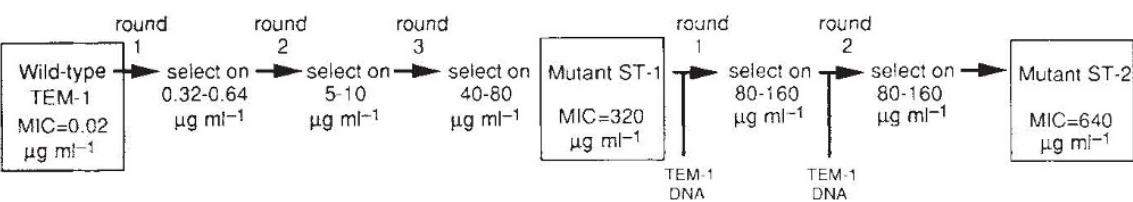

was due to chromosomal mutations. DNA sequencing showed that both mutants had 9 single-base-pair mutations.
7. Arkin, A. \& Youvan, D. C. Proc. natn. Acad. Sci. U.S.A. 89, 7811-7815 (1992).

8. Delagrave, S. \& Youvan, D. C. Biotechnology 11, 1548-1552 (1993).

9. Palzkill, T. \& Botstein, D. J. Bact. 174, 5237-5243 (1992).

10. Oliphant, A. R., Nussbaum, A. L. \& Struhl, K. Gene 44, 177-183 (1986).

11. Hermes, J. D. Blacklow, S. C. \& Knowles, J. R. Proc natn. Acad. Sci. U.S.A. 87, 696-700 (1990).

12. Leung, D. W., Chen, E. \& Goeddel, D. V. Technique 1, 11-15 (1989)

13. Caldwell, R. C. \& Joyce, G. F. PCR Meth. Applic. 2, 28-33 (1992).

14. Kauffman, S. A. The Origins of Order (Oxford Univ. Press, New York, 1993).

15. Kauffman, S. A. J. theor. Biol. 157, 1-7 (1992)

16. Bartel, D. P. \& Szostak, J. W. Science 261, 1411-1418 (1993)
17. Tuerk, C. \& Gold, L. Science 249, 505-510 (1990).

18. Joyce, G. F. Scient. Am. 267, 90-97 (1992).

19. Jacoby, G. A. \& Medeiros, A. A. Antimicrob. Ag. Chemother. 35, 1697-1704 (1991)

20. Collatz, E., Labia, R. \& Gutmann, L. Molec. Microbiol. 4, 1615-1620 (1990)

21. Philippon, A., Labia, R. \& Jacoby, G. Antimicrob. Ag. Chemother. 33, 1131-1136 (1989) 22. McCafferty, J. Griffiths, A. D., Winter, G. \& Chiswell, D. J. Nature 348, 552-554 (1990).

23. Huse, W. D., Sastry, L., Iverson, S. A. \& Kang. A. S. Science 246, 1275-1278 (1989).

23. Huse, W. D., Sastry, L., Iverson, S. A. \&

25. Ambler, R. P. et al. Biochem. J. 276, 269-272 (1991).

ACKNOWLEDGEMENTS. We thank A. Crameri for assistance with DNA sequencing, P. Schatz for suggesting the model system and $W$. Dower for valuable discussions.

ERRATA

Parthenogenetic activation of oocytes in

\section{c-mos-deficient mice}

\section{Naohiro Hashimoto, Nobumoto Watanabe, Yasuhide Furuta, Hiroyuki Tamemoto, Noriyuiki Sagata, Minesuke Yokoyama, Kenji Okazaki, Mariko Nagayoshi, Naoki Takeda, Yoji Ikawa \& Shinichi Alzawa}

Nature 370, 68-71 (1994)

FIGURE $3 b$ and $c$ of this Letter was an early version that should not have been published. The correct version of this figure is shown here.
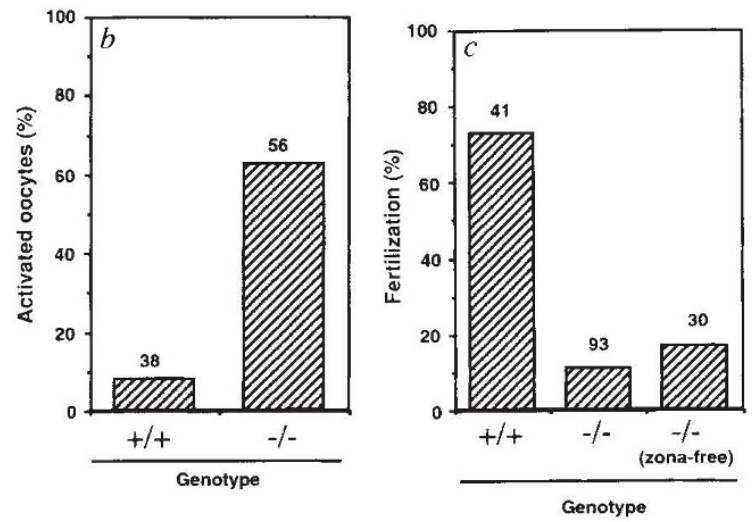

Genotype
Degradation of trifluoroacetate in oxic and anoxic sediments

\author{
Pleter T. Visscher, Charles W. Culbertson \\ \& Ronald S. Oremland
}

Nature 369, 729-731 (1994)

IN the last sentence of the opening paragraph of this Letter, an error was introduced during editing in which fluoroform was referred to as a "potential ozone-depleting compound." In fact, fluoroform as well as other HFCs were recently shown by Ravishankara et al. ' to have "negligibly small" ozone depletion potentials.

1. Ravishankara, A. R. et al. Science 263, 71-75 (1994).

\section{Miller-Dieker lissencephaly gene encodes a subunit of brain platelet-activating factor acetylhydrolase}

\section{Mitsuharu Hattori, Hideki Adachi, Masafumi Tsujimoto, Hiroyuki Arai \& Keizo Inoue}

Nature 370, 216-218 (1994)

THE word 'acetylhydrolase' was accidentally omitted from the end of the title of this paper. The correct title should read "Miller-Dieker lissencephaly gene encodes a subunit of brain platelet-activating factor acetylhydrolase". 